\title{
HII Regions in Resonance-Ring Spiral Galaxies
}

\author{
Deborah A. Crocker, Pamela D. Baugus ${ }^{1}$, and R. Buta \\ Dept. of Physics and Astronomy, Box 870324, University of Alabama, \\ Tuscaloosa, AL 35487
}

\section{Introduction}

Resonance ring galaxies are barred (and sometimes nonbarred) spiral galaxies which display an inner, outer, or nuclear ring in the light distribution. This study is of the HII region properties in 32 probable resonance ring galaxies selected mainly from the Catalog of Southern Ringed Galaxies (CSRG; Buta 1995). The objective of the study is to illustrate the distributions of HII regions in classic examples of ringed galaxies, and to relate the observed properties to resonance theory. Here we present a brief summary of a more extensive paper on the results of this study (Crocker, Baugus, \& Buta 1996).

\section{Observations}

The data consist of calibrated $\mathrm{H} \alpha(+[\mathrm{NII}])$ line and local continuum images obtained in 1993 August with the CTIO 1.5-m telescope and a TEK 1024 CCD array. Types represented in the sample cover the range $\mathrm{S}^{+}$to $\mathrm{Sc}$, and both barred and nonbarred galaxies are included. The sample focuses mainly on early-to-intermediate Hubble type systems having outer pseudorings which belong to the subclasses known as $R_{1}, R_{1}^{\prime}$, and $R_{2}^{\prime}$ (see Buta, these proceedings), based on blue-light images.

\section{Results}

There is a great deal of individual variation in the HII region properties of our sample galaxies, as one might surmise. There are a number of properties which they share and several cases have characteristics worth noting. These are:

- a general prominence of the observed rings, particularly inner rings, in $H \alpha$

- a distinct lack of HII regions in the bar regions of SB(r) galaxies

- a distinctive $\mathrm{H} \alpha$ morphology in NGC 6782 consisting of a circular nuclear ring, a pointy oval inner ring, and a partial outer pseudoring

- that the most intrinsically oval inner rings in the sample have HII regions more concentrated and more luminous near the intrinsic ring major axis (see Figure 1)

\footnotetext{
${ }^{1}$ also Rhodes College, 2000 North Parkway, Memphis, TN 38112
} 

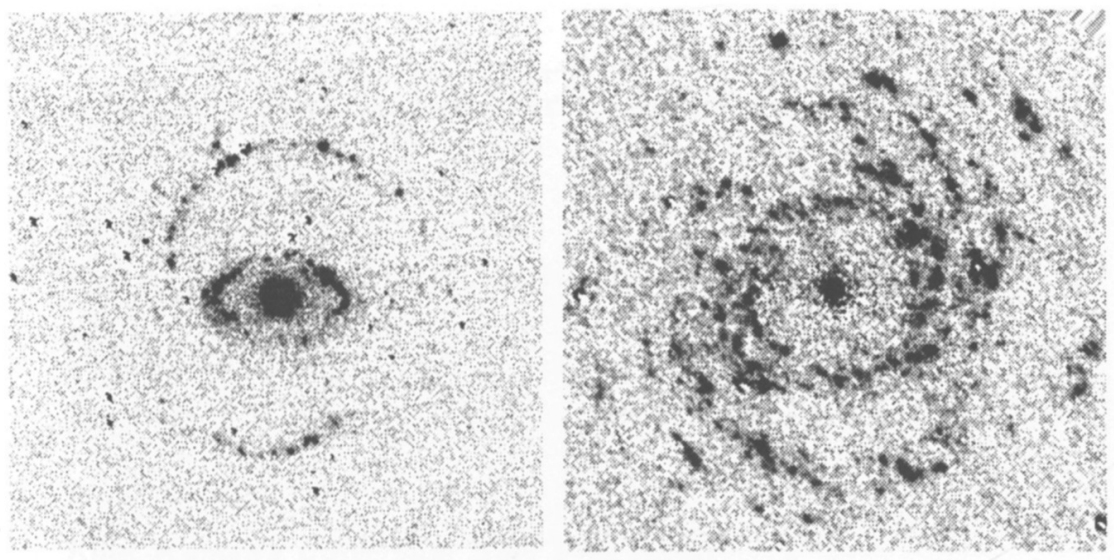

Figure 1. Distributions of HII regions in barred galaxy inner rings of different intrinsic shapes: NGC 6782 (left), an example of an extremely oval inner ring, and NGC 7329 (right), an example of a nearly circular inner ring. Both images are deprojected according to parameters in Crocker, Baugus, \& Buta (1996) and have been rotated so that the bar axis is horizontal.

- a double nuclear ring/pseudoring in NGC 1317

- that the outer HII regions in some galaxies tend to follow an $\mathrm{R}_{2}^{\prime}$ morphology even when the stellar distribution shows a strong $\mathrm{R}_{1}$ morphology

\section{Discussion}

The characteristics we find in these galaxies are consistent with some expectations of resonance theory. The appearance of a double nuclear ring feature in NGC 1317 can be explained in terms of an inner Lindblad resonance (ILR). The inner nuclear ring is aligned nearly perpendicular to the primary bar/oval and may be connected to the $x_{2}$ family of periodic orbits. The outer nuclear ring is nearly aligned parallel to the primary bar/oval and may be connected to periodic orbits of the $x_{1}$ family located just outside the outer ILR of a double ILR.

The HII characteristics of some of the outer rings fit into an evolutionary scenario whereby outer rings develop initially as $R_{1}$ types and evolve into $R_{2}^{\prime}$ types (Byrd et al. 1994). This is seen in NGC 1326, NGC 6782, IC 1438, and UGC 12646.

Our study confirms earlier findings of the tendency for inner rings to be prominent in $\mathrm{H} \alpha$ and for the bar regions in early-type $\mathrm{SB}(\mathrm{r})$ systems to be mostly devoid of HII regions (Peterson et al. 1976; Buta 1988 and references therein; Ryder \& Dopita 1993; Phillips, these proceedings; Garcia-Barreto, these proceedings). 


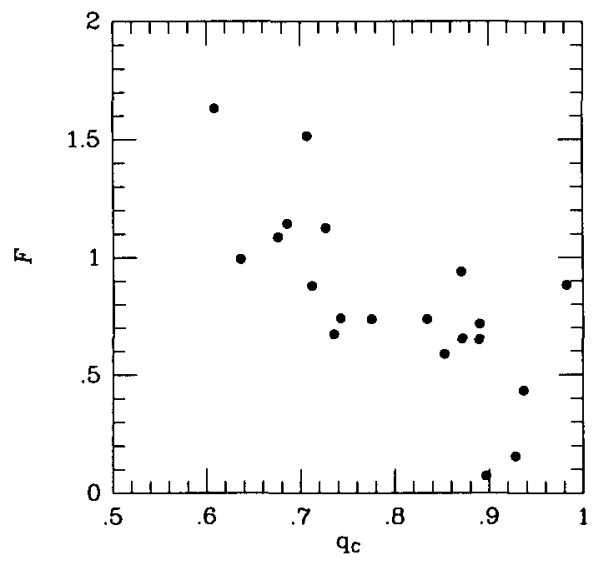

Figure 2. The behavior of the $\mathrm{H} \alpha$ intensity around inner rings versus the inner ring axial ratio as determined through Fourier analysis.

\subsection{Azimuthal Distribution of HII Regions}

Our main finding from the study is a definite correlation between the distribution of HII regions around an inner ring and the intrinsic shape of the ring. Extremely oval inner rings have the HII regions "bunched up" near the intrinsic ring major axis (also the bar axis, as in NGC 6782, Figure 1, left), while nearly circular rings have a more even distribution of HII regions (as in NGC 7329, Figure 1, right). Through the use of the relative $2 \theta$ Fourier amplitude, this effect is quantified in Figure 2 for 18 galaxies whose deprojected continuum ring axis ratios $\left(q_{C}\right)$ range from 0.60 to nearly 1.0 . As $q_{C}$ decreases, the relative $2 \theta$ amplitude $F$ increases. The dynamical implication of this result is that gas gathered into the rings moves along oval streamlines aligned with the bar axis, and that the material slows down in the rotating reference frame at the ring major axis. This argues that inner rings lie within the corotation resonance (Contopoulos 1979).

Acknowledgments. Support for this paper was provided by NSF grants EHR-9108761, AST-9014137 and NSF REU grant AST 9300413.

\section{References}

Buta, R. 1988, ApJS, 66, 233

Buta, R. 1995, ApJS, 96, 39

Byrd, G. et al 1994, AJ, 108, 476

Crocker, D. A, Baugus, P., and Buta, R., 1996, ApJS, in press

Contopoulos, G. 1979, in Photometry, Kinematics, and Dynamics of Galaxies, D. S. Evans, Austin: Univ. Texas Press, 425

Peterson, C., Rubin, V., Ford, W., and Thonnard, N. 1976, ApJ, 208, 662

Ryder, S. \& Dopita, M. 1993, ApJS, 88415 